\title{
FURIN correlated with immune infiltration serves as a potential biomarker in SARS-CoV-2 infection- related lung adenocarcinoma
}

\section{Lianxiang Luo ( $\sim$ luolianxiang321@gdmu.edu.cn )}

Guangdong Medical College Zhanjiang Campus: Guangdong Medical University https://orcid.org/0000-0002-3391-9713

\section{Manshan Li}

Guangdong Medical College Zhanjiang Campus: Guangdong Medical University Jiating Su

Guangdong Medical College Zhanjiang Campus: Guangdong Medical University

\section{Xinyue Yao}

Guangdong Medical College Zhanjiang Campus: Guangdong Medical University

Hui Luo

Guangdong Medical College Zhanjiang Campus: Guangdong Medical University

\section{Research Article}

Keywords: FURIN, Immune infiltration, Lung adenocarcinoma, SARS-CoV-2, Biomarker

Posted Date: August 30th, 2021

DOI: https://doi.org/10.21203/rs.3.rs-629424/v2

License: (c) (1) This work is licensed under a Creative Commons Attribution 4.0 International License. Read Full License 


\section{Abstract}

FURIN, as a proprotein convertase, has been found to be expressed in a variety of cancers and plays an important role in cancer. In addition, SARS-CoV-2 (severe acute respiratory syndrome coronavirus 2) requires FURIN to enter human cells. However, the role of FURIN in lung adenocarcinoma remains unclear. And the expression of SARS-CoV-2 related gene in lung adenocarcinoma has not been clarified. Therefore, in order to explore the prognostic value and mechanism of FURIN in lung adenocarcinoma, we performed bioinformatics analysis with Oncomine, TIMER (Tumor Immune Estimation Resource), GEPIA (Gene Expression Profiling Interactive Analysis), HPA (human protein atlas), UALCAN, PrognoScan, Kaplan-Meier plotter, cBioPortal, and LinkedOmics databases. And then We used GSE44274 in the GEO (Gene Expression Omnibus) database to analyze the expression of FURIN in LUAD patients who infected with SARS-CoV. FURIN was highly expressed in lung adenocarcinoma and was significantly associated with poor overall survival. FURIN expression was found to be correlated with six major permeable immune cells and with macrophage immune marker in LUAD patients. In addition, SARS-CoV- 2 infection might affect the expression of FURIN. FURIN can be used as a promising biomarker for determining prognosis and immune infiltration in LUAD patients.

\section{Introduction}

Lung cancer has become the most common type of cancer and the leading cause of cancer death among men and women worldwide, according to the latest cancer statistics[1]. Lung cancer is divided into nonsmall cell lung cancer and small cell lung cancer, in which non-small cells can be divided into large cell carcinoma, squamous cell carcinoma and adenocarcinoma. And lung adenocarcinoma is one of the cancers that seriously threaten human health. Non-small cell lung cancer accounts for about $85 \%$ of lung cancer and is found to be mostly in the middle and late stages, with a low five-year survival rate [2]. At present, targeted treatment can't get better prognostic effect due to its drug resistance. Traditional chemotherapy and surgery also do not have good postoperative effect[3].Therefore, we need to look for reliable biomarkers for diagnosis and prognosis of LUAD, which will contribute to the effective treatment of LUAD.

FURIN is an important member of pre-protein processing enzymes. FURIN is expressed by the FUR (FES upstream region) gene on chromosome 15 , high in the salivary glands, liver, bone marrow, and relatively low in muscle cells[4]. According to literature, abnormal expression or activation of FURIN can promote the formation and development of a variety of malignant tumors, including colon carcinoma, rabies, head and neck cancers, lung cancer, skin cancer and brain tumors. And FURIN has been confirmed to be expressed in breast cancer, squamous cell carcinoma and transverse muscular tumors, as well as studies have found high levels of FURIN genes in lung adenocarcinoma. In addition, FURIN has an important role in immunity [4-6]. The article of Guo etc. [7] built a based on ten new prognostic immune related genes signal, can be used to predict the prognosis of lung adenocarcinoma, including FURIN. What's more, FURIN is also known as the main switch for tumor growth and progression[7, 8]. And there could be a relationship between FURIN activities and the different clinical scenarios of the 2019-nCoV 
infection[9]. According to the literature, we know that SARS-CoV-2 enters human cells by binding to ACE2, and then the S protein is cut open by TMPRSS2, which then, with the help of FURIN, promotes the virus to enter human cells[10, 11]. Moreover, there are multiple FURIN bits in the S protein of SARS-CoV-2, and the involvement of FURIN in the S protein startup process may increase the likelihood that SARS-CoV-2 will enter the cell[12]. This prompted us to explore the link between FURIN and SARS-CoV-2 in patients with lung adenocarcinoma.

COVID-19 is spreading around the world, and cancer patients are thought to be more susceptible to SARSCoV-2[13]. According to the literature, we understand that SARS-CoV-2 requires ACE2, FURIN to enter human cells[10,14]. Based on the study of ACE2 in COVID-19 patients with Uterine Corpus Endometrial Carcinoma and Kidney Renal Papillary Cell Carcinoma[15]. And FURIN expressed strong findings in the lungs[16]. Therefore, it is of great significance to understand the associations between FURIN and patients with COVID-19 complicated with lung adenocarcinoma.

Based on the findings of these studies, we chose FURIN to investigate immune immersion with lung adenocarcinoma to explore the immune mechanism between FURIN and lung adenocarcinoma. To better explore the role of FURIN in LUAD, in this study, we first explored the differential expression of FURIN in different organizations using Oncomine, GEPIA and other databases. Meanwhile, the Human Protein Atlas database (HPA) was used to detect FURIN expression in normal and tumor tissues. Moreover, we used UALCAN database to analyze the expression of FURIN in different clinical feature subgroups. PrognoScan databases and Kaplan-Meier plotter were used to assess the relationship between FURIN and prognosis of LUAD patients comprehensively. Then we used the cBioPortal database to determine the type and frequency of FURIN changes in LUAD patients. Tumor immunoassay resource (TIMER) database was used to further investigate the association between FURIN expression levels and immune cell infiltration, different immune cell subsets markers in LUAD. And UALCAN database was used to analyze the promoter methylation level of FURIN in LUAD and the correlation between FURIN expression level and different subgroups of LUAD patients. Finally, we used LinkedOmics database to evaluate the gene co-expression of FURIN in LUAD and to investigate their role in tumor immunity. Our results provided novel insight into the function of FURIN in LUAD, and a theoretical basis for the early diagnosis, prognosis and targeted therapy of LUAD. In addition, high expression of FURIN lung adenocarcinoma patients were more likely to be infected with SARS-CoV-2.

\section{Methods}

\section{Oncomine Database Analysis.}

Oncomine (https://www.oncomine.org/) database is a web-based data mining platform with a microarray database of most human cancers [17]. This study used tumor database to analyze the expression level of FURIN in LUAD. In this study, we searched according to criteria: (A) analysis type: cancer and normal tissue; (B) data type: mRNA; (C) threshold: P-VALUE: 0.01, FOLD CHANGE: All, GENE RANK: All. 


\section{TIMER Database Analysis.}

This study used the TIMER (https://cistrome.shinyapps.io/timer/) database which provides a userfriendly web interface for dynamic analysis and visualization of some associations[18] to analyze the expression of FURIN and immune cells (B-cells, CD4-T cells, CD8-T cells, neutral granulocytes, macrophages, and degenerative cells) in LUAD patients. At the same time, the correlation between FURIN expression and the genetic markers of tumor infiltrating immune cells was also discussed.

\section{GEPIA Database Analysis.}

GEPIA (http://gepia.cancer-pku.cn/) is a web-based tool to deliver fast and customizable functionalities based on TCGA and GTEx data[19]. This study analyzed the expression of FURIN in tumors and normal tissues.

\section{UALCAN Database Analysis.}

In this study, we analyzed the characteristics of tumors and normal samples in a single clinical pathology phase, as well as the relative expression of different genes in the tumor subtype group, using UALCAN (http://ualcan.path.uab.edu) which uses TCGA level 3 RNA-seq and clinical data from 31 cancer types[20].

\section{Kaplan-Meier plotter Database Analysis.}

Kaplan-Meier plotter[21] (http://kmplot.com/analysis/) provides a way to explore the survival effects of multiple genes on patients with 21 different types of cancer, including breast cancer $(n=6,234)$, ovarian cancer $(n=2,190)$, lung cancer $(n=3,452)$ and stomach cancer $(n=1,440)$ groups with larger sample sizes. Therefore, we used this database to explore the relationship between FURIN expression and results in LUAD patients, and analyze clinical pathological factors and the effect of FURIN on LUAD patient outcomes.

\section{Human Protein Atlas Database Analysis.}

HPA (https://www.proteinatlas.org/) is an integrated database of human cell lines, cancer tissue types, and human tissue types using a variety of Omics technologies that provides expression maps of 32 human tissues and their proteins, and uses antibody analysis to accurately evaluate protein positioning[22]. Therefore, we use this database to analyze protein expression and immunologic tissue chemistry (IHC) in FURIN in normal tissues and LUAD tissues.

\section{PrognoScan Database Analysis}

PrognoScan (http://gibk21.bse.kyutech.ac.jp/PrognoScan/index.html)[23]aims to facilitate the analysis of gene prognostic values by comparing the relationship between gene expression and related results, including the overall survival rate (OS) of various published cancer microarray datasets. Therefore, we use this database to evaluate the relationship between FURIN expression and patient outcomes. 


\section{cBioProtal Database Analysis.}

cBioProtal (https://www.cbioportal.org) is an open-access resource for interactive exploration of multidimensional cancer genomics data sets, currently containing 225 cancer studies[24]. We used cBioPortal to analyze FURIN alterations in the LUAD sample.

\section{LinkedOmics Database Analysis.}

LinkedOmics (http://www.linkedomics.org/) is a web-based platform for analyzing 32 TCGA cancerassociated multi-dimensional datasets[25]. We use LinkedOmics database to analyze FURIN coexpression, presenting in volcano plots, heat maps.

\section{GeneMANIA Database Analysis.}

GeneMANIA (http://genemaia.org) website is used to construct the PPI network through a large number of correlation data including physical interactions, co-expression, predicted, co-localization, pathway, genetic interactions, and shared protein domains[26]. We used GeneMANIA database to construct PPI network of FURIN.

\section{Data Analysis.}

The gene expression profiles of GSE44274 were downloaded from the GEO database. We extracted the expression of FURIN to analyze its different expression in the control group and treatment group by using the $\mathrm{R}$ language.

\section{Results}

\section{Expression of FURIN in lung adenocarcinoma}

We used Oncomine and TIMER to analyze the expression of FURIN mRNA in different cancer tissues and normal tissues. Among many cancers, FURIN had a higher expression in breast, stomach, head and neck, kidney, ovarian, and lung cancer, but FURIN had a lower expression in esophageal cancer, leukemia, lymphoma, melanoma, and myeloma (Figure 1A). At the same time, we also used Oncomine to analyze tumors in LUAD and normal samples, which showed that FURIN expression in LUAD was higher than normal lung gland tissue in different patient data sets (FURIN expressed in Selamat, Hou data sets) (Figure 1D, E). Analysis in the TIMER database showed that FURIN expression differences in tumor tissue and adjacent normal tissues (Figure 1B): FURIN expression in COAD(colon cancer), KIRC(kidney renal clear cell carcinoma),KIRP(kidney renal papillary cell carcinoma) was significantly lower in adjacent normal tissue; in contrast, FURIN expression in BLCA(road cortical cancer), ESCA(esophageal cancer), HNSC(head and neck squamous cell carcinoma), LUAD (lung adenocarcinoma), STAD(stomach cancer), UCEC(endometrial cancer) were higher than those of neighboring tissues. Data mining was further confirmed using the GEPIA and UALCAN databases. The GEPIA analysis showed a slight increase in expression of FURIN in LUAD tissue compared to normal lung gland tissue $(P<0.05)$ (Figure $1 C)$. On the 
other hand, UALCAN analyzed the protein expression of FURIN and found that its expression in tumor tissue was slightly higher than that of normal tissue (Figure 1F). In addition, we also used HPA to analyze IHC, and Figure $1 \mathrm{G}$ showed that FURIN significantly reduced protein expression in normal tissue and significantly increased protein expression in tumor tissue. According to different analysis results of FURIN above, FURIN was slightly higher in LUAD, indicating that the abnormal expression of FURIN may be closely related to the development, transfer and prognosis of LUAD.

\section{Distribution of FURIN expression in clinical characteristics sub-groups}

In order to study whether FURIN expression in lung adenocarcinoma patients associated with clinical factors, we used the UALCAN database to detect the distribution of FURIN in different histological subtypes of lung adenocarcinoma, it was found that there was statistical significance between FURIN expression and the first three tumor stages $(P<0.05)$ and an increase in the distribution of FURIN in the first three tumor stages (Figure 2A). At the same time, FURIN was expressed higher in TP53 in a nonmutant state (Figure 2B). Analysis of gender, age, subtypes of different tissues and different lymph node metastasis showed that the expression level of FURIN in patients with lung adenocarcinoma increased relative to normal samples (Figure 2C-F). In addition, FURIN was significantly higher in women than in men in lung adenocarcinoma (Figure 2C). And smokers had a higher expression of FURIN than nonsmokers (Figure 2D).

\section{Prognosis analysis of FURIN expression in LUAD}

Next, we used the PrognoScan database to explore the relationship between FURIN expression and LUAD patient prognostics. The study found a significant correlation between prognosis in LUAD patients and expression of FURIN $(P<0.05)$ (Figure 3A, B). The higher FURIN expression was associated with a poorer LUAD prognosis (OS HR=1.26, COX P-VALUE $=0.000641$; RFS HR=0.69, COX P-

VALUE $=0.023887$ ). Moreover, there are other types of cancer that showed some correlation between prognostic and FURIN expression, such as breast and ovarian cancer (Figure 3C, D). We then conducted a meta-analysis of the results generated from different data sets in PrognoScan database. Meta-analysis showed that the combined $\mathrm{HR}$ and $95 \% \mathrm{Cl}$ of OS associated with high FURIN expression were $1.08(0.98,1.18)$, but heterogeneity was observed in 21 data sets $\left(I^{\wedge} 2=55.2 \%, P=0.001\right)$ (Figure 3E).

Then, we used the Kaplan-Meier plotter to further validate the prognosis of FURIN expression. Patients were divided into two groups based on the median expression of each group of FURIN. It is found the increased expression of FURIN was associated with the prognosis of LUAD patients $(P<0.05)$, and overall survival (OS) and first progression (FP) were also highly affected by the increased expression of FURIN mRNA (OS:HR=1.67, Log-rank $P=1.4 \mathrm{e}-05$; $F P: H R=1.62$, Log-rank $P=0.0024$ ) (Figure 3F, $G$ ), suggesting that FURIN may be a reliable biomarker for the prognosis of LUAD.

\section{LUAD patients with FURIN mutation frequency, type and FURIN changed LUAD patient survival analysis}


Based on sequencing data from LUAD patients in the TCGA database, we used the cBioPortal database to determine the type and frequency of FURIN changes in LUAD patients. FURIN changed in 30 out of 503 patients (6\%) (Figure 4A). These changes were 5 mutations $(0.99 \%), 3(0.6 \%)$ amplification, $1(0.2 \%)$ deep deletion and 20 (3.98\%) mRNA high, and 1 multiple change (0.2\%). Therefore, mRNA high is the most common type of LUAD. The types, sites and case number of the FURIN genetic alteration are further presented in Figure 4B. We found that missense mutation is the most common type of FURIN change. In addition, we explored the potential link between FURIN gene changes and clinical survival prognostics in LUAD patients. Figure 4C, D, E shows that patients without FURIN change showed better prognosis in Overall survival rate $(P=1.636 \mathrm{E}-3)$, progression-free survival rate $(P=6.679 \mathrm{E}-4)$ and disease-specific survival rate $(P=2.380 E-3)$ compared to patients with FURIN changes.

\section{Correlation expression and correlation analysis of immune cell penetration in LUAD}

Then, we used the TIMER database to determine whether the high expression of FURIN in LUAD tissue was associated with six major permeable immune cells (B cells, CD4+T cells, CD8+T cells, neutrophils, macrophages, and dendritic cells). The results showed that FURIN expression levels were associated with $B$ cells $(r=-0.144, p=1.46 e-03)$, CD8+T cells $(r=-0.157, p=4.92 e-04), C D 4+T$ cells $(r=-0.039, p=3.97 e-02)$, macrophages cells $(r=-0.108, p=1.77 e-02)$, neutrophil cells $(r=-0.124, p=6.28 e-03)$, dendritic cells $(r=-0.243$, $p=5.64 e-08)$ related. As showed in the scatter chart, the expression level of FURIN was negatively related to most immune cells, indicating that the expression level of FURIN was negatively related to LUAD immune infiltration. To further study the relationship between immune cellular immersion and FURIN expression in LUAD, we used the TIMER database to further map Kalan-Meier to assess the prognostic value of each of the six immune cells (Figure $5 B$ ). We found that the expression of $B$ cells (Log-rank $P=0$ ) and dendritic cells (Log-rank $P=0.048$ ) were associated with the prognosis of LUAD, indicating that FURIN plays an important role in regulating immune cell penetration.

\section{Correlation analysis between FURIN mRNA levels and different subgroup markers of immune cells}

Next, based on a set of immune markers in LUAD, we used the TIMER database to further explore the link between FURIN expression and immune cell penetration levels. Specifically, we evaluated the correlation between FURIN expression and Parker levels for a particular subset of cells (including CD8+T cells, B cells, T cells, monocytes, TAM, neutral granulocytes). At the same time, we analyzed different subsets of T cells, namely Th1, Th2, Tfh, Th17, Treg, T cell exhaustion. As a result of tumor purity adjustment results in clinical samples, the results showed that the expression of FURIN in LUAD tissue was significantly related to the expression of most marker genes in immune filtration cells (Table 1).

The study found that the expression of FURIN in LUAD was significantly related to the expression of immune marker genes in CD8+T cells, T cells, monocytes, TAM, and dendritic cells. In particular, CD8A, CD8B of CD8+T cell, CD3D, CD2 of T cells, CD86, CSF1R of Monocyte, CCL2 of TAM cells, ITGAM of Neutrophils, HLA-DPB1, HLA-DQB1, HLA-DRA, HLA-DPA1, CD1C of Dendritic cells are all closely related to LUAD's FURIN level $(P<0.0001)$. In addition, we found that the expression of FURIN is closely related to 
Th2, Tfh, Th17, T cell exhaustion (e.g. STAT6, ICOS, STAT3, HAVCR2), further confirming the relationship between FURIN expression and LUAD immune infiltration.

Then we used the TIMER 2.0 database and used different algorithms to further explore the relationship between macrophages and FURIN expression, and found that FURIN expression was negatively related to macrophage immersion levels (Figure 6A, B). The expression of FURIN was also related to the polarization of macrophages, and the expression of FURIN was negatively related to the polarization of M1 macrophages and M2 macrophages (Figures 6C, D, E, F). In addition, we found that the expression of FURIN was positively related to markers of M1 macrophages and negatively related to the markers of M2 macrophages (Figure 6G, H). The expression of FURIN in lung adenocarcinoma is associated with macrophage polarization, indicating that FURIN may affect the immune response through polarized macrophages.

\section{FURIN promoter methylation level analysis in LUAD}

After analysis, we observed an increase in FURIN expression in LUAD, so we will further study the reasons for the increase in FURIN expression. Because methylation is closely related to disease progression, low methylation can lead to genomic instability and may activate related genes, all of which we use the UALCAN database to verify the methylation levels of FURIN promoters in LUAD. As showed in Figure 7A, the level of methylation of the promoter of FURIN in normal tissues was slightly higher than that of LUAD. In addition, group analysis of promoter methylation was carried out according to the patient's sex, tumor stage, smoking habits, lymph node metastasis, and age. The results showed that the promoter methylation was associated with sex, tumor stage, smoking habits and age in LUAD patients (Figure 7B, $C, D, F)$, but there was no correlation between promoter methylation and LUAD patients with N3's lymph node metastasis. These results showed that lower promoter methylation may lead to high expression levels of FURIN in LUAD.

\section{Construction of gene co-expression network and gene enrichment analysis}

To better understand the biological processes of FURIN in LUAD, we used the LinkedOmics database to analyze differences in FURIN co-expression genes. As showed in Figure 8A, 9000 genes (red dots) are positively correlation with FURIN and 10988 genes (green dots) are negatively correlation $(P<0.05)$. As showed in Figure 8B-C, the first 50 positive and negative genes associated with FURIN are shown as heat maps. FURIN expression is presented with genes strong positive correlation such as INSL4 (positive correlation, $r=0.618, p=1.13 E-55)$, CPS1 ( $r=0.597, p=4.37 E-51)$, C6orf176 ( $r=0.594, p=1.33 E-50)$, but negative correlation with genes such as STK17A $(r=0.469, p=1.24 E-29), D H D H(r=0.447, p=1.12 E-26)$, CNN3 ( $r=0.446, p=1,31 \mathrm{E}-26)$. What's more, we used GeneMANIA to construct PPI network of FURIN.As showed Figure8D, NOTCH3, GPC3, PROZ, GDF11, FLNA, GAS6, SERPINB8, LECT1, EDA, F7, PCSK5, PACS1, F10, PCSK1, PROS1, SORL1, MMP7, NGF, PCSK2, F9 interact with FURIN. After that, we used DAVID to perform Gene Ontology (GO) enrichment analysis based on the FURIN co-expression gene network. As showed in Figure 8E, According to $\mathrm{GO}$ enrichment analysis, FURIN co-expression genes were mainly concentrated in biological process including the positive regulation of substrate adhesion- 
dependent cell spreading, blood coagulation, fibrin clot formation, signal transduction, plasminogen activation, positive regulation of heterotypic cell-cell adhesion, CAMP catabolic process, cell cycle, cell protein complex assembly, fibrinolysis, positive regulation of exocytosis, positive regulation of transcription, DNA-templated. At the cell composition level, FURIN co-expression is mainly enrich in extracellular exosomes, and FURIN co-expression in molecular function level were mainly focused on metal ion binding (Figure 8F).

\section{SARS-CoV infection may affect the expression of FURIN}

The SARS-CoV-2 S protein has multiple FURIN bits. And FURIN can help the virus enter human cells[12]. In addition, FURIN plays an important role in lung adenocarcinoma. Therefore, it is crucial to analyze the expression of FURIN in LUAD patients who infected with SARS-CoV. We used GSE44274 in the GEO database for research. GSE44274 includes four biological sample types of mouse lung tissue (SARSmus, UV-V, Lung; SARSmus, UV-V+TLR, Lung; SARSmus, PBS, Lung and SAESmus, HKU, Lung). Based on whether the samples infected with SARS-CoV, we divided it into treatment group and control group, which were used to analyze the expression of FURIN in mice lung infected with SARS-CoV-2. As showed in the figure, the FURIN level in the treatment group was significantly lower than that in the control group. The results showed statistical significance, which indicated that SARS-CoV-2 infection may affect the expression of FURIN (Figure 9).

\section{Discussion}

This study found that FURIN was expressed stronger in LUAD tumor tissue and was significantly related to its prognosis. At the same time, the correlation between FURIN expression and prognosis based on different clinical characteristics suggested that FURIN may be a potential independent biomarker of LUAD prognosis. In addition, we studied the mutation type and frequency of FURIN and found that the missense mutation is the most important type of change in FURIN. Then, we analyzed the correlation between FURIN and immune infiltration, as well as between FURIN and different subsets of immune cells. Studies have showed that FURIN is associated with six large osmotic immune cells and has a stronger effect on the penetration of B cells and Dendritic cells. Therefore, penetration of FURIN in B cells and Dendritic cells may be one of the prognostic factors. We then analyzed the effects of FURIN expression on the promotion of methylation. Finally, we studied FURIN co-expression analysis and GO enrichment analysis.

We found that FURIN expression levels were higher in the first three tumor stages (stage 1, 2, 3), and FURIN had the highest expression in stage 3, which expressed a possible relationship between FURIN's primary involvement in LUAD stage 3 , and there may be a relationship between FURIN expression and LUAD disease outcomes. Therefore, we used the PrognoScan database and its related data for survival analysis, and found that high expression of FURIN may be related to the adverse prognosis of LUAD. In addition, the analysis of Kaplan-Meier plotter showed a correlation between FURIN expression levels and different clinical characteristics of LUAD, further indicating that FURIN is an independent risk factor for 
LUAD. Therefore, FURIN deserves further clinical validation as a potential diagnostic and prognosis marker of LUAD.

It is worth noting that the expression of FURIN has a significant correlation with the state of TP53 mutation, the expression of FURIN in LUAD patients without TP53 mutation is higher. TP53 protein on a variety of cell stress response, regulate the expression of the target gene, thereby inducing cell cycle arrest, apoptosis, senescence, DNA repair or metabolic changes. At the same time, we found that FURIN and its co-expression genes are also mainly enriched in CAMP decomposition metabolic processes, cell cycles, cell protein complex assembly, etc. of the biological process level. In addition, we used the cBioPortal database to explore the types and frequencies of FURIN changes, and found that missense mutations are the main types of FURIN changes, and that mRNA level high are the main types of lung adenocarcinoma cases, and found a potential link between FURIN gene changes and clinical survival prognostication in LUAD patients.

Our study also found that FURIN expression levels were closely correlated to the penetration of B cells and Dendritic cells. There is a certain correlation between B cells and LUAD prognostics, as well as between Dendritic cells and LUAD prognostics. These findings showed that $B$ cells and Dendritic cells may be key factors with prognostic values in FURIN. In addition, we have found in the literature that Dendritic cell plays a central role in both immune response and adaptive response, and that rapid loss of Dendritic cell function can delay T-cell immune response in patients with COVID-19[27]. According to our findings, FURIN is negatively associated with Dendritic cell, and high expression of FURIN in patients with lung adenocarcinoma may lead to a decrease in Dendritic cell. And our study found a negative correlation between B cells and highly expressed FURIN, which is associated with higher expression of lung adenocarcinoma. Therefore, the decrease in B cells in lung adenocarcinoma may indicate susceptible to COVID-19 in patients with lung adenocarcinoma. This is consistent with the reduction of lymphocytes as one of the clinical characteristics of COVID-19[28]. In addition, we understand that DC vaccine therapy can be used to combat the COVID-19 epidemic and to overcome the possibility of re-infection of the virus[29]. At the same time, in our study of correlations between FURIN and several immune characteristics, we found that most marker genes in immune infiltration cells were also associated with highly expressed FURIN. Among them, the marker genes of Dendritic cells are associated with FURIN (HLA-DPB1, HLA-DQB1, HLA-DRA, HLA-DPA1, CD1C are significantly correlated with FURIN, $P<0.0001$ ). The correlation between FURIN and Dendritic cells and their associated marker genes is important for prognostication in LUAD patients, but FURIN may be a key factor in the treatment of Dendritic cells that require further study. In addition, macrophages include M1 macrophages and M2 macrophages. M1 macrophages have a proinflammatory function and they can attract immune cells to the infected site. M2 macrophages have anti-inflammatory function and they can reduce inflammation or heal wounds[30]. The expression of FURIN in lung adenocarcinoma is associated with macrophage polarization, indicating that FURIN may affect the immune response through polarized macrophages.

To study the reasons for the increase in FURIN in LUAD, we studied the methylation level of FURIN in LUAD and found that the level of methylation of promoters in LUAD decreased. As a result, FURIN may be 
activated and regulated by its low methylation, explaining the degree of promotion of FURIN in LUAD. Since then, in gene co-expression analysis, we have seen that the expression of INSL4, CPS1, C6orf176 have the strongest positive correlation with the expression of FURIN, while the expression of STK17A, $\mathrm{DHDH}$, and CNN3 have the strongest negative correlation with the expression of FURIN. According to the relevant literature, we known that INSL4, which is positively associated with FURIN, is a member of the restricted expression insulin/IGF/relaxation super family in the placenta. In addition, INSL4 was previously found through in-body selection in the invasive breast cancer cell subclone, and it has also been found to be a new target downstream of LKB1 defects. At the same time, INSL4 signals are considered to have a potentially important role in the inactivation of non-small cell lung cancer in LKB1[31]. CPS1 is the first speed-limiting mitochondrial enzyme in the urea cycle, and if the mutation leads to a lack of CPS1, it can lead to life-threatening hyperammonemia. Moreover, the decrease of CPS1 will lead to the accumulation of ammonia and the reduction of nucleic acid synthesis pathways, resulting in the growth of cancer cells[32]. C6orf176 is a new regulator of camp-mediated gene expression, involved in the regulation of camp signaling path. Moreover, C6orf176 plays an important role in the occurrence and development of various diseases such as tumors, and is considered to be a potential target for diagnosis and treatment of diseases[33]. STK17A, which is negatively associated with FURIN, is one of the direct and DNA damage-induced p53 target genes involved in cellular processes[34]. Calponin 3 (CNN3) is a member of the behavioral-binding molecular family in the CNN family[35]. It works in regulating neural tube morphology in embryonic development, as well as in cell differentiation, proliferation, and migration through stress fiber formation or cell bone remodeling[36, 37]. As a result, when FURIN is expressed, the body may regulate biological processes by affecting the expression of positive and negative genes, which can affect the condition in patients with lung adenocarcinoma.

According to the study, we known that FURIN, as one of the proteins in the transformation enzyme family, plays a vital role in the maturation of viral glycoproteins, and that FURIN activates the glycoproteins of coronavirus[38]. We found that SARS-CoV-2 enters human cells by binding to ACE2, after which the virus enters cells with the help of FURIN[10,11]. Because FURIN is strongly expressed in the lungs[16], coronavirus can enter cells with the help of FURIN. This suggests that SARS-CoV-2 is highly likely to infect lung cancer patients with high expression FURIN. At the same time, our research showed that SARS-CoV affected the expression of FURIN.

\section{Conclusion}

This study provided comprehensive evidence for the value of FURIN in lung cancer progression and its potential as a biological target and prognostic predictor of LUAD. Our findings suggested that upregulation of FURIN in LUAD predicts adverse outcomes in the overall survival of patients, possibly due to multiple physiological processes affecting FURIN expression. Furthermore, we found a significant correlation between FURIN and most immune features. However, the analysis in this study was based on servers or databases, which may differ in specific experiments. It is important to validate the results of the analysis through experiments in our future research. 


\section{Declarations}

\section{Funding}

This project was supported by the PhD Start-up Fund of Guangdong Medical University (B2019016); Administration of Traditional Chinese Medicine of Guangdong Province (20201180); Administration of Traditional Chinese Medicine of Guangdong Province (20211223); Science and Technology Special Project of Zhanjiang (2019A01009); Basic and Applied Basic Research Program of Guangdong Province (2019A1515110201); Key Program of Marine Economy Development(Six Marine Industries) Special Foundation of Department of Natural Resources of Guangdong Province(GDNRC[2020]038); Fund of Southern Marine Science and Engineering GuangdongLaboratory (Zhanjiang) (ZJW-2019-007). Discipline Construction Project of Guangdong Medical University (4SG21004G).

\section{Conflicts of Interest}

The authors declare that they have no conflicts of interest in this study.

\section{Authorship contributions}

Conceptualization, Lianxiang Luo; Data curation, Manshan Li; Formal analysis, Jiating Su; Funding acquisition, Lianxiang Luo; Investigation, Manshan Li, Xinyue Yao; Project administration, Lianxiang Luo and Hui Luo; Resources, Hui Luo; Visualization, Jiating Su and Xinyue Yao; Writing - original draft, Lianxiang Luo and Manshan Li; Writing - review \& editing, Lianxiang Luo.

\section{References}

1 Bray F, Ferlay J, Soerjomataram I, et al. Global cancer statistics 2018: GLOBOCAN estimates of incidence and mortality worldwide for 36 cancers in 185 countries. CA: A Cancer Journal for Clinicians. 2018;68(6):394-424.

2 Li L, Sun Y, Feng M, Wang L, Liu J. Clinical significance of blood-based miRNAs as biomarkers of nonsmall cell lung cancer. Oncol Lett. 2018;15(6):8915-8925.

3 Wei X, Lai Y, Li J, et al. PSCA and MUC1 in non-small-cell lung cancer as targets of chimeric antigen receptor T cells. Oncoimmunology. 2017;6(3):e1284722.

4 Braun E, Sauter D. Furin-mediated protein processing in infectious diseases and cancer. Clin Transl Immunology. 2019;8(8):e1073.

5 Maria Vähätupa1, Saara Aittomäki2,6, Zuzet Martinez Cordova2,6, Ulrike May1, Stuart Prince1, Hannele Uusitalo-Järvinen3, Tero A Järvinen1,4,7, Marko Pesu2,5,7. T-cell-expressed proprotein convertase FURIN inhibits DMBA/TPA-induced skin cancer development. 
6 Jaaks P, Bernasconi M. The proprotein convertase furin in tumour progression. Int J Cancer. 2017;141(4):654-663.

7 Bassi D E, Fu J, Lopez de Cicco R, Klein-Szanto A J. Proprotein convertases: "master switches" in the regulation of tumor growth and progression. Mol Carcinog. 2005;44(3):151-61.

8 Klein-Szanto A J, Bassi D E. Proprotein convertase inhibition: Paralyzing the cell's master switches. Biochem Pharmacol. 2017;140(8-15.

9 Usul Afsar C. 2019-nCoV-SARS-CoV-2 (COVID-19) infection: Crucıalıty of Furın and relevance with cancer. Med Hypotheses. 2020;140(109770.

10 Coutard B, Valle C, de Lamballerie X, et al. The spike glycoprotein of the new coronavirus 2019-nCoV contains a furin-like cleavage site absent in CoV of the same clade. Antiviral Res. 2020;176(104742.

11 Lukassen S, Chua R L, Trefzer T, et al. SARS-CoV-2 receptor ACE2 and TMPRSS2 are primarily expressed in bronchial transient secretory cells. Embo j. 2020;39(10):e105114.

12 Drak Alsibai K. Expression of angiotensin-converting enzyme 2 and proteases in COVID-19 patients: A potential role of cellular FURIN in the pathogenesis of SARS-CoV-2. Med Hypotheses. 2020;143(109893.

13 Dai M, Liu D, Liu M, et al. Patients with Cancer Appear More Vulnerable to SARS-CoV-2: A Multicenter Study during the COVID-19 Outbreak. Cancer Discov. 2020;10(6):783-791.

14 Shang J, Wan Y, Luo C, et al. Cell entry mechanisms of SARS-CoV-2. Proc Natl Acad Sci U S A. 2020;117(21):11727-11734.

15 Yang J, Li H, Hu S, Zhou Y. ACE2 correlated with immune infiltration serves as a prognostic biomarker in endometrial carcinoma and renal papillary cell carcinoma: implication for COVID-19. Aging (Albany NY). 2020;12(8):6518-6535.

16 Mbikay M, Sirois F, Yao J, Seidah N G, Chrétien M. Comparative analysis of expression of the proprotein convertases furin, PACE4, PC1 and PC2 in human lung tumours. $\mathrm{Br} \mathrm{J}$ Cancer. 1997;75(10):1509-14.

17 Rhodes D R, Yu J, Shanker K, et al. ONCOMINE: a cancer microarray database and integrated datamining platform. Neoplasia. 2004;6(1):1-6.

$18 \mathrm{Li} \mathrm{T}$, Fan J, Wang B, et al. TIMER: A Web Server for Comprehensive Analysis of Tumor-Infiltrating Immune Cells. Cancer Res. 2017;77(21):e108-e110.

19 Tang Z, Li C, Kang B, et al. GEPIA: a web server for cancer and normal gene expression profiling and interactive analyses. Nucleic Acids Res. 2017;45(W1):W98-w102. 
20 Chandrashekar D S, Bashel B, Balasubramanya S A H, et al. UALCAN: A Portal for Facilitating Tumor Subgroup Gene Expression and Survival Analyses. Neoplasia. 2017;19(8):649-658.

21 Lánczky A, Nagy Á, Bottai G, et al. miRpower: a web-tool to validate survival-associated miRNAs utilizing expression data from 2178 breast cancer patients. Breast Cancer Res Treat. 2016;160(3):439446.

22 Chiang S C, Han C L, Yu K H, Chen Y J, Wu K P. Prioritization of cancer marker candidates based on the immunohistochemistry staining images deposited in the human protein atlas. PLoS One. 2013;8(11):e81079.

23 Mizuno H, Kitada K, Nakai K, Sarai A. PrognoScan: a new database for meta-analysis of the prognostic value of genes. BMC Med Genomics. 2009;2(18.

24 Gao J, Aksoy B A, Dogrusoz U, et al. Integrative analysis of complex cancer genomics and clinical profiles using the cBioPortal. Sci Signal. 2013;6(269):pl1.

25 Vasaikar S V, Straub P, Wang J, Zhang B. LinkedOmics: analyzing multi-omics data within and across 32 cancer types. Nucleic Acids Res. 2018;46(D1):D956-d963.

26 Warde-Farley D, Donaldson S L, Comes O, et al. The GeneMANIA prediction server: biological network integration for gene prioritization and predicting gene function. Nucleic Acids Res. 2010;38(Web Server issue):W214-20.

27 Zhou R, To K K, Wong Y C, et al. Acute SARS-CoV-2 Infection Impairs Dendritic Cell and T Cell Responses. Immunity. 2020;53(4):864-877.e5.

28 Huang C, Wang Y, Li X, et al. Clinical features of patients infected with 2019 novel coronavirus in Wuhan, China. Lancet. 2020;395(10223):497-506.

29 Saadeldin M K, Abdel-Aziz A K, Abdellatif A. Dendritic cell vaccine immunotherapy; the beginning of the end of cancer and COVID-19. A hypothesis. Med Hypotheses. 2021;146(110365.

30 Murray P J, Allen J E, Biswas S K, et al. Macrophage activation and polarization: nomenclature and experimental guidelines. Immunity. 2014;41(1):14-20.

$31 \mathrm{Ma} \mathrm{C}$, Luo H, Cao J, et al. Identification of a Novel Tumor Microenvironment-Associated Eight-Gene Signature for Prognosis Prediction in Lung Adenocarcinoma. Front Mol Biosci. 2020;7(571641.

$32 \mathrm{Wu}$ G, Zhao Z, Yan Y, et al. CPS1 expression and its prognostic significance in lung adenocarcinoma. Annals of Translational Medicine. 2020;8(6):341.

33 Reitmair A, Sachs G, Im W B, Wheeler L. C6orf176: a novel possible regulator of cAMP-mediated gene expression. Physiol Genomics. 2012;44(2):152-61. 
34 Wei W, Liu C. Prognostic and predictive roles of microRNA-411 and its target STK17A in evaluating radiotherapy efficacy and their effects on cell migration and invasion via the p53 signaling pathway in cervical cancer. Mol Med Rep. 2020;21(1):267-281.

35 Junghans D, Herzog S. Cnn3 regulates neural tube morphogenesis and neuronal stem cell properties. The FEBS Journal. 2018;285(2):325-338.

36 Shibukawa Y, Yamazaki N, Daimon E, Wada Y. Rock-dependent calponin 3 phosphorylation regulates myoblast fusion. Exp Cell Res. 2013;319(5):633-48.

37 Shibukawa Y, Yamazaki N, Kumasawa K, et al. Calponin 3 regulates actin cytoskeleton rearrangement in trophoblastic cell fusion. Mol Biol Cell. 2010;21(22):3973-84.

$38 \mathrm{Li} \mathrm{W}$, Moore M J, Vasilieva N, et al. Angiotensin-converting enzyme 2 is a functional receptor for the SARS coronavirus. Nature. 2003;426(6965):450-4.

\section{Tables}

Table 1. Correlation analysis between FURIN and relate genes and markers of immune cells in TIMER. 


\begin{tabular}{|c|c|c|c|c|c|}
\hline \multirow[t]{3}{*}{ Description } & \multirow[t]{3}{*}{ Gene markers } & \multicolumn{3}{|c|}{ LUAD } & \\
\hline & & \multicolumn{2}{|c|}{ None } & \multicolumn{2}{|l|}{ Purity } \\
\hline & & Cor & P Value & Cor & P Value \\
\hline \multirow[t]{2}{*}{ CD8+T cell } & CD8A & -0.151 & 0.000752 & -0.174 & $7.50 \mathrm{E}-05$ \\
\hline & CD8B & -0.168 & $1.81 \mathrm{E}-04$ & -0.185 & $2.52 \mathrm{E}-05$ \\
\hline \multirow[t]{2}{*}{ B cell } & MS4A1 & -0.066 & 1.41E-01 & -0.1 & 2.40E-02 \\
\hline & FCRL2 & -0.141 & 1.73E-03 & -0.162 & $2.26 \mathrm{E}-04$ \\
\hline \multirow[t]{3}{*}{ T cell } & CD3D & -0.167 & 1.99E-04 & -0.187 & 1.97E-05 \\
\hline & CD3E & -0.117 & $9.05 \mathrm{E}-03$ & -0.145 & $9.65 \mathrm{E}-04$ \\
\hline & CD2 & -0.18 & $6.07 \mathrm{E}-05$ & -0.202 & $3.89 \mathrm{E}-06$ \\
\hline \multirow[t]{2}{*}{ Monocyte } & CD86 & -0.244 & 3.95E-08 & -0.259 & 2.90E-09 \\
\hline & CSF1R & -0.178 & $6.92 \mathrm{E}-05$ & -0.204 & $3.16 \mathrm{E}-06$ \\
\hline \multirow[t]{2}{*}{ TAM } & CCL2 & -0.224 & 4.95E-07 & -0.237 & $6.13 \mathrm{E}-08$ \\
\hline & IL10 & -0.161 & $3.21 \mathrm{E}-04$ & -0.183 & 3.03E-05 \\
\hline Neutrophil & ITGAM & -0.166 & 2.17E-04 & -0.194 & 9.27E-06 \\
\hline \multirow[t]{5}{*}{ Dendritic cell } & HLA-DPB1 & -0.324 & $1.70 \mathrm{E}-13$ & -0.329 & 1.97E-14 \\
\hline & HLA-DQB1 & -0.236 & 1.10E-07 & -0.253 & 5.79E-09 \\
\hline & HLA-DRA & -0.403 & $1.02 E-20$ & -0.397 & 0 \\
\hline & HLA-DPA1 & -0.356 & $3.27 \mathrm{E}-16$ & -0.361 & $1.75 \mathrm{E}-17$ \\
\hline & CD1C & -0.218 & $1.01 \mathrm{E}-06$ & -0.233 & $9.25 \mathrm{E}-08$ \\
\hline \multirow[t]{3}{*}{ Th1 } & TBX21 & -0.096 & 3.35E-02 & -0.129 & $3.43 \mathrm{E}-03$ \\
\hline & STAT4 & -0.146 & $1.11 \mathrm{E}-03$ & -0.166 & 1.60E-04 \\
\hline & IFNG & -0.107 & 1.78E-02 & -0.139 & 1.57E-03 \\
\hline \multirow[t]{3}{*}{ Th2 } & STAT6 & 0.175 & $9.21 \mathrm{E}-05$ & 0.174 & 7.51E-05 \\
\hline & STAT5A & -0.13 & 3.95E-03 & -0.16 & 2.77E-04 \\
\hline & IL13 & -0.102 & 2.34E-02 & -0.118 & 7.52E-03 \\
\hline \multirow[t]{3}{*}{ Tfh } & BCL6 & 0.114 & $1.12 \mathrm{E}-02$ & 0.109 & 1.29E-02 \\
\hline & ICOS & -0.197 & 1.10E-05 & -0.214 & $9.84 \mathrm{E}-07$ \\
\hline & CXCL13 & -0.142 & $1.52 \mathrm{E}-03$ & -0.159 & 2.87E-04 \\
\hline Th17 & STAT3 & -0.298 & $1.48 \mathrm{E}-11$ & 0.293 & $1.21 \mathrm{E}-11$ \\
\hline
\end{tabular}




\begin{tabular}{|llllll||} 
Treg & STAT5B & 0.148 & $9.97 \mathrm{E}-04$ & 0.13 & $3.05 \mathrm{E}-03$ \\
& TGFB1 & -0.093 & $4.00 \mathrm{E}-02$ & -0.116 & $8.59 \mathrm{E}-03$ \\
& CTLA4 & -0.12 & $7.78 \mathrm{E}-03$ & -0.152 & $5.62 \mathrm{E}-04$ \\
& LAG3 & -0.084 & $6.18 \mathrm{E}-02$ & -0.117 & $7.68 \mathrm{E}-03$ \\
& HAVCR2 & -0.252 & $1.38 \mathrm{E}-08$ & -0.265 & $1.09 \mathrm{E}-09$ \\
\hline
\end{tabular}

LUAD: Lung Adenocarcinoma; TAM: tumor-associated macrophage; Th: T helper cell; Tfh: Follicular helper T cell; Treg: regulatory T cell; Cor: $\mathrm{R}$ value of Spearman's correlation; none: correlation without adjustment; purity: correlation adjusted by purity.

\section{Figures}

A FURIN

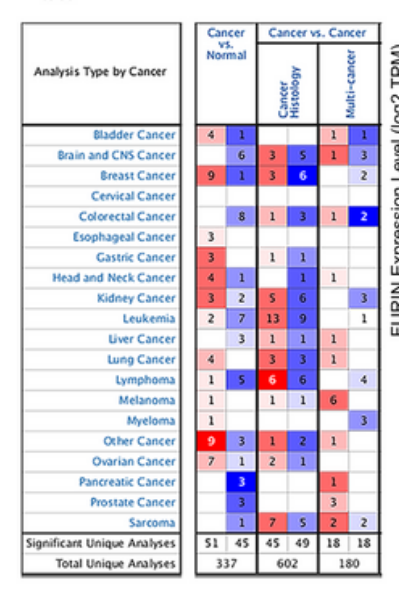

D FURIN Expression in Selamat Lung Lung adenocarcinoma vs. Normal

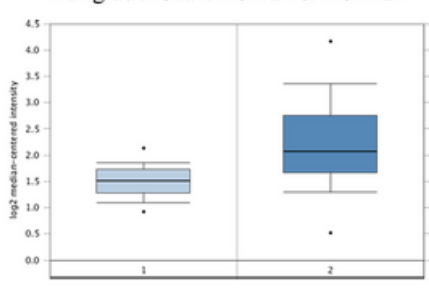

P-value:3.68E-8

t-Test:6.019

Fold Change: 1.600
B

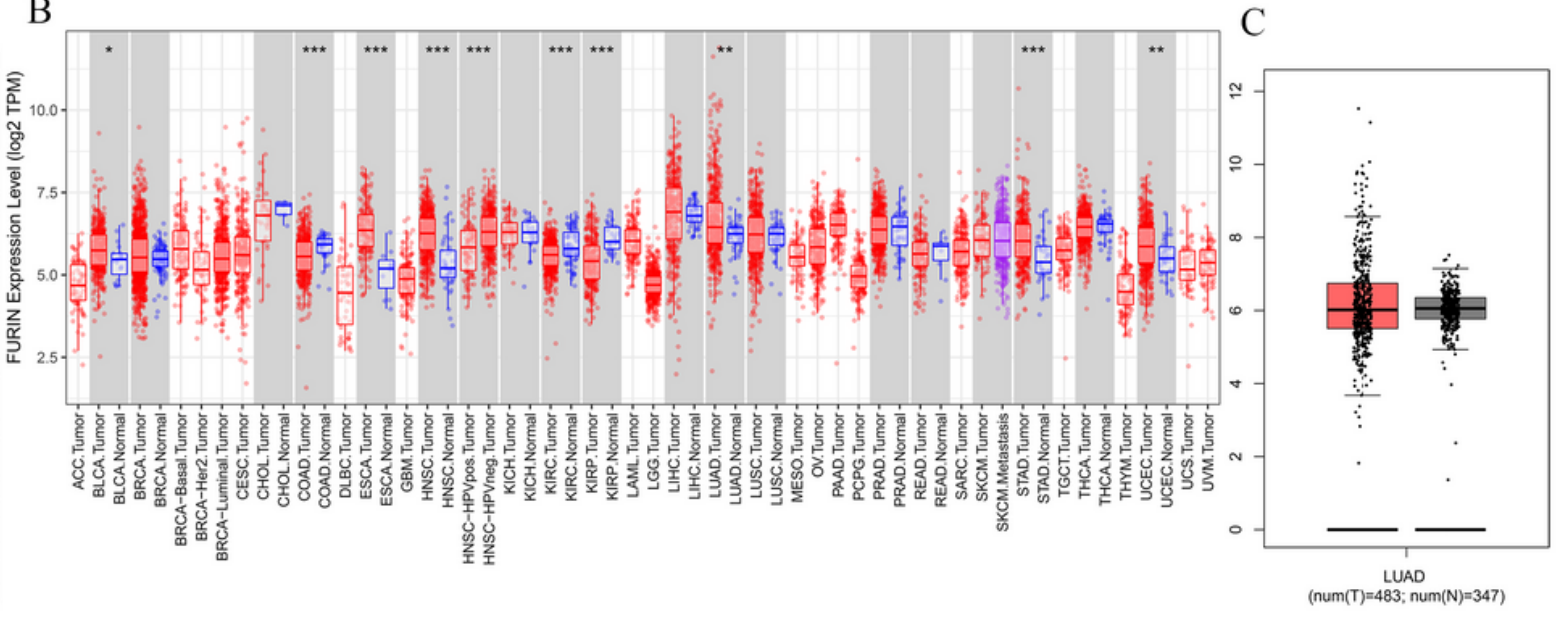

E FURIN Expression in Hou Lung F Lung adenocarcinoma vs. Normal

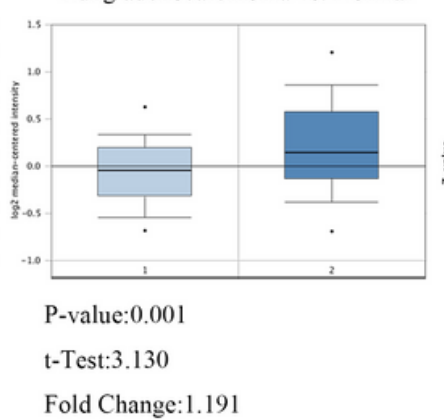

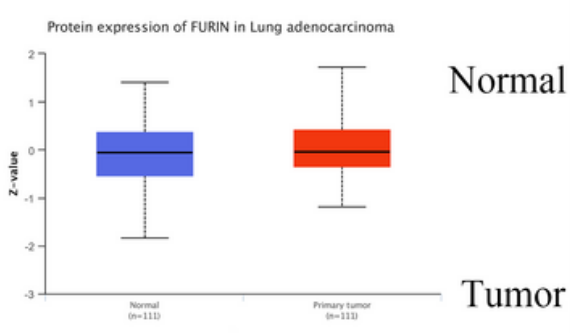

G

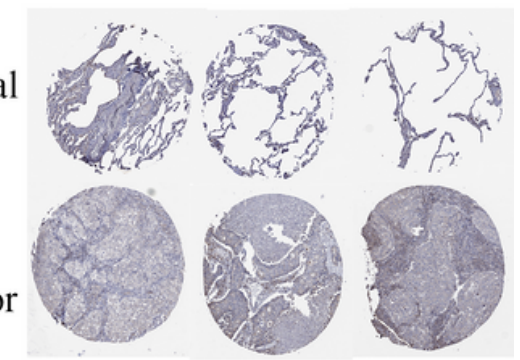

Figure 1

Expression of FURIN in lung adenocarcinoma. (A) Expression of FURIN in different types of tumor tissue and normal sample tissue in the Oncomine database. (B) The expression of FURIN in different types of tumor tissue and normal sample tissue in the TIMER database. (C) GEPIA database showed that the mRNA expression level of FURIN in LUAD was slightly higher than that of normal tissue. (D-E) FURIN in different patient data sets in Oncomine was expressed higher than normal in lung adenocarcinoma. (F) In 
the UALCAN database, the protein expression of FURIN in lung adenocarcinoma $(n=111)$ and the protein expression of normal tissue $(n=111)$. (G) Expression in HPA in the expression of lung adenocarcinoma and normal tissue.

A Expression of FURIN in LUAD based on individual cancer

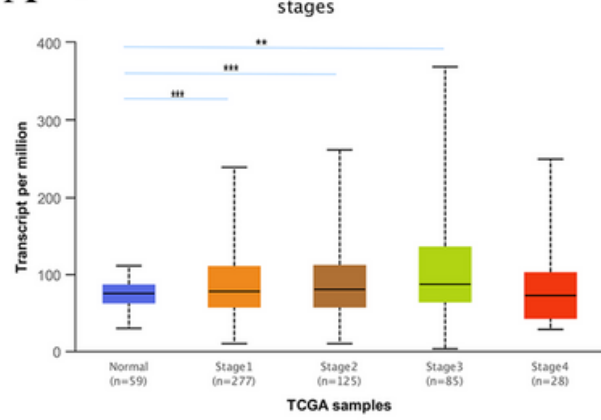

D Expression of FURIN in LUAD based on patient's smoking

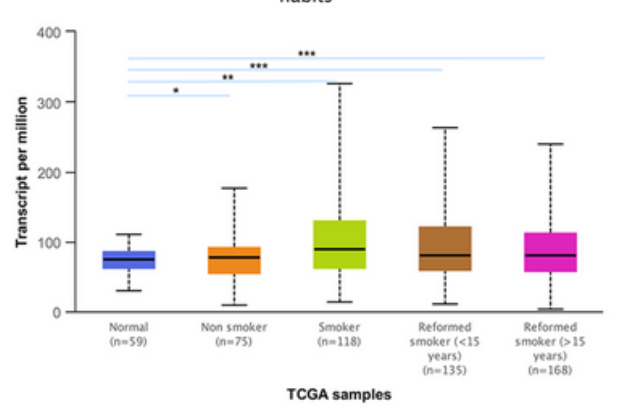

B Expression of FURIN in LUAD based on TP53 muation status

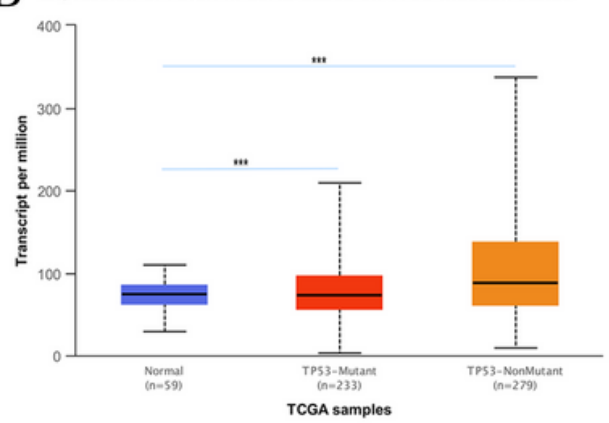

E Expression of FURIN in LUAD based on Histological subtypes

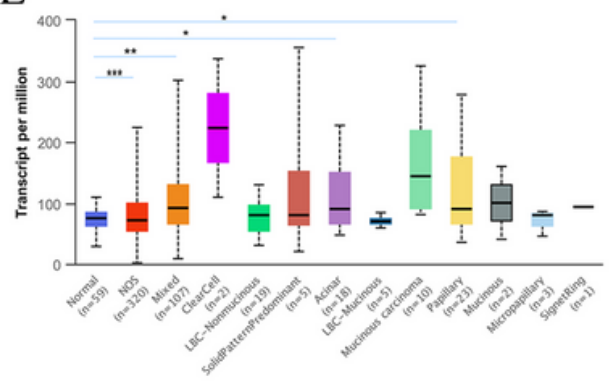

TCGA samples
C Expression of FURIN in LUAD based on patient's gender

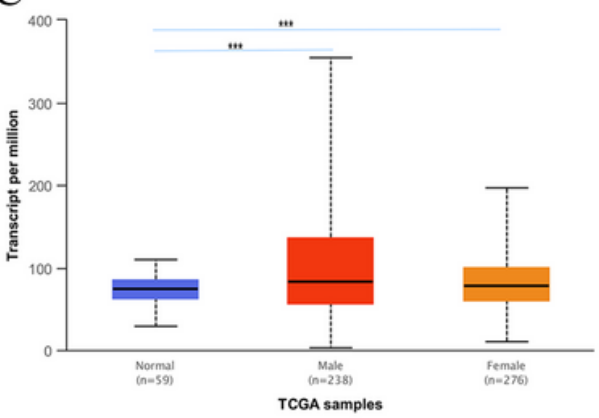

F Expression of FURIN in LUAD based on nodal metastasis

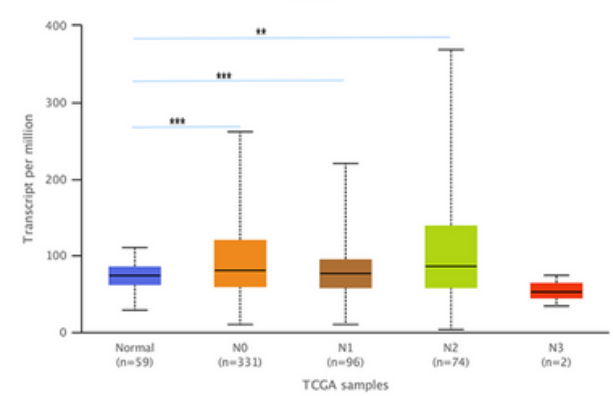

Figure 2

Expression of FURIN in clinically characterized sub-groups. (A) FURIN is based on the expression of a single stage of cancer in LUAD. (B) FURIN is based on the expression of the TP53 mutation state in LUAD. (C) FURIN is expressed in LUAD based on the patient's gender. (D) FURIN is based on the expression of the patient's smoking habits in LUAD. (E) FURIN is based on the expression of histological subtypes in LUAD. (F) FURIN is based on the expression of node metastasis in LUAD. 
A Luad os

HR:1.26 COX P-VALUE:0.000641

Kaplan-Meier plot

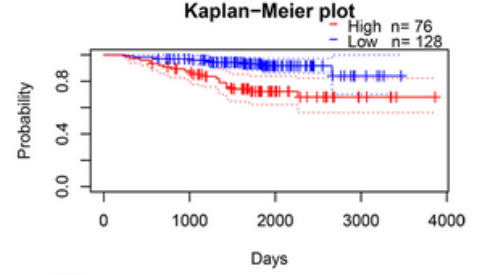

E
B LUADRFS

HR:0.69 COX P-VALUE:0.023887

Kaplan-Meier plot

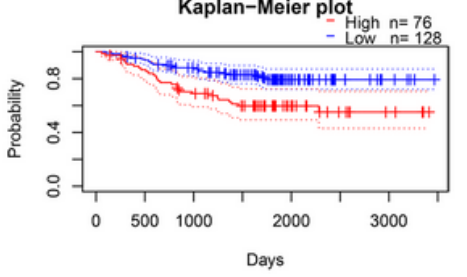

C BRCA RFS

HR:-0.17 COX P-VALUE:0.200192

Kaplan-Meier plot ${ }_{\text {tigh }} n=166$

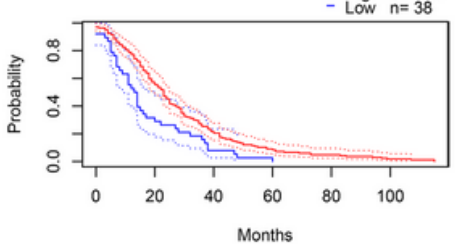

D OV OS

HR:0.42 COX P-VALUE:0.042909

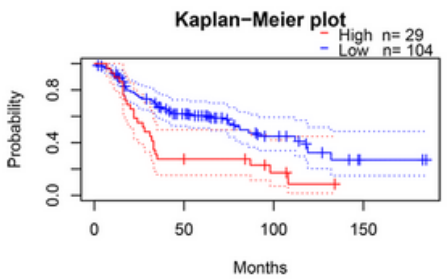

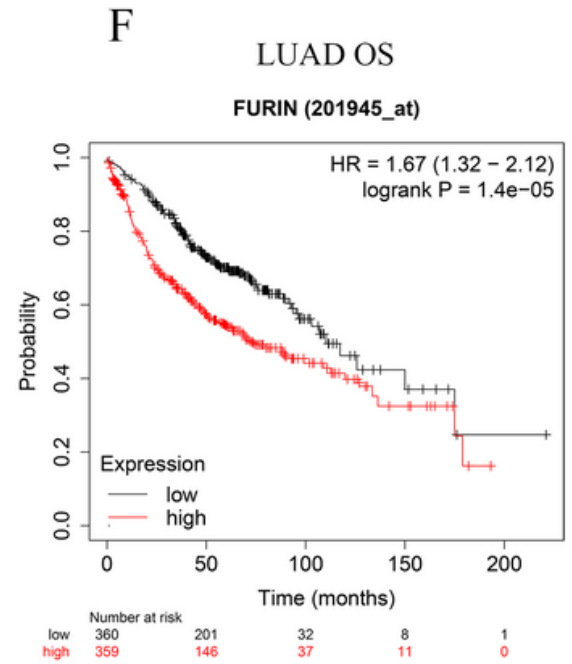

G

LUAD FP

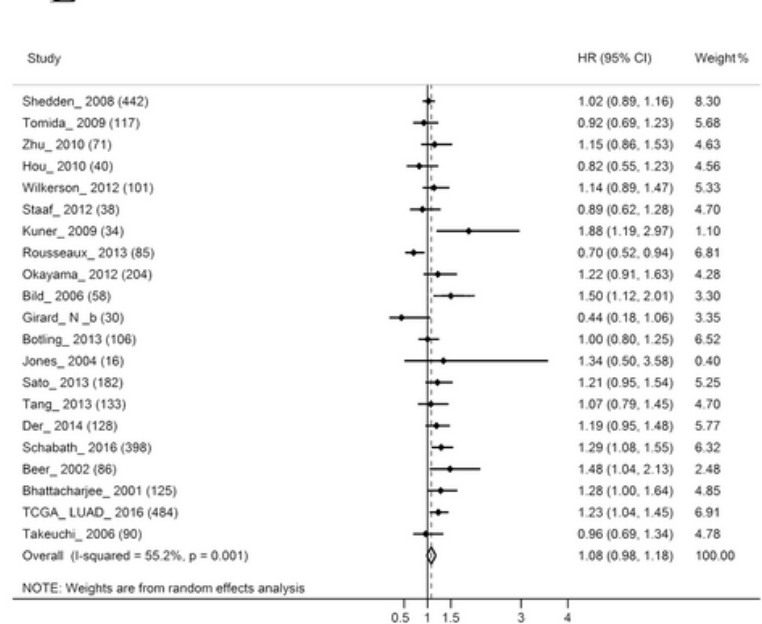

FURIN (201945_at)

HOU_ 2010 (40)

Wikerson_ 2012 (101)

Staat_2012 (38)

d_ 2006 (5\%)

Boting_ 2013 (100)

Schabath_2016 (390)

Shattachariee_2001 (125)

NOTE: Weights are from

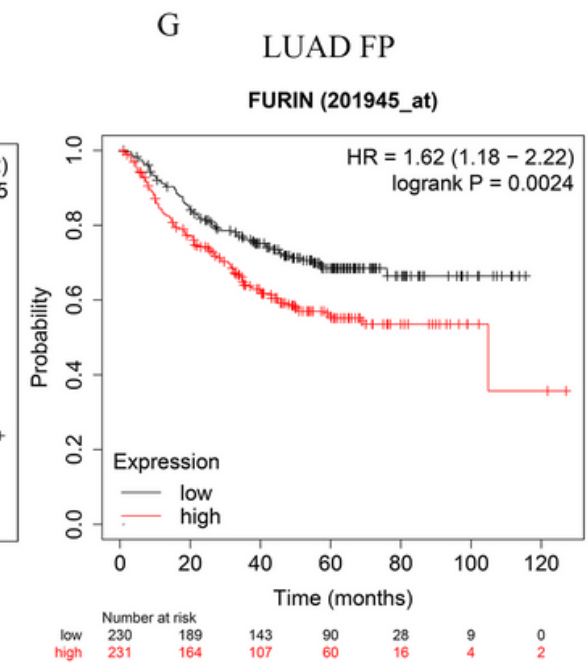

\section{Figure 3}

FURIN is associated with survival results. (A-B) Prognostics in LUAD patients were significantly related to the expression of FURIN. (C-D) Association of prognostic expression with FURIN expression in patients with other types of cancer. (E) Total survival rate (OS) of LUAD in FURIN gene expression. (F) first progression survival rate (FP) of LUAD in FURIN gene expression. 


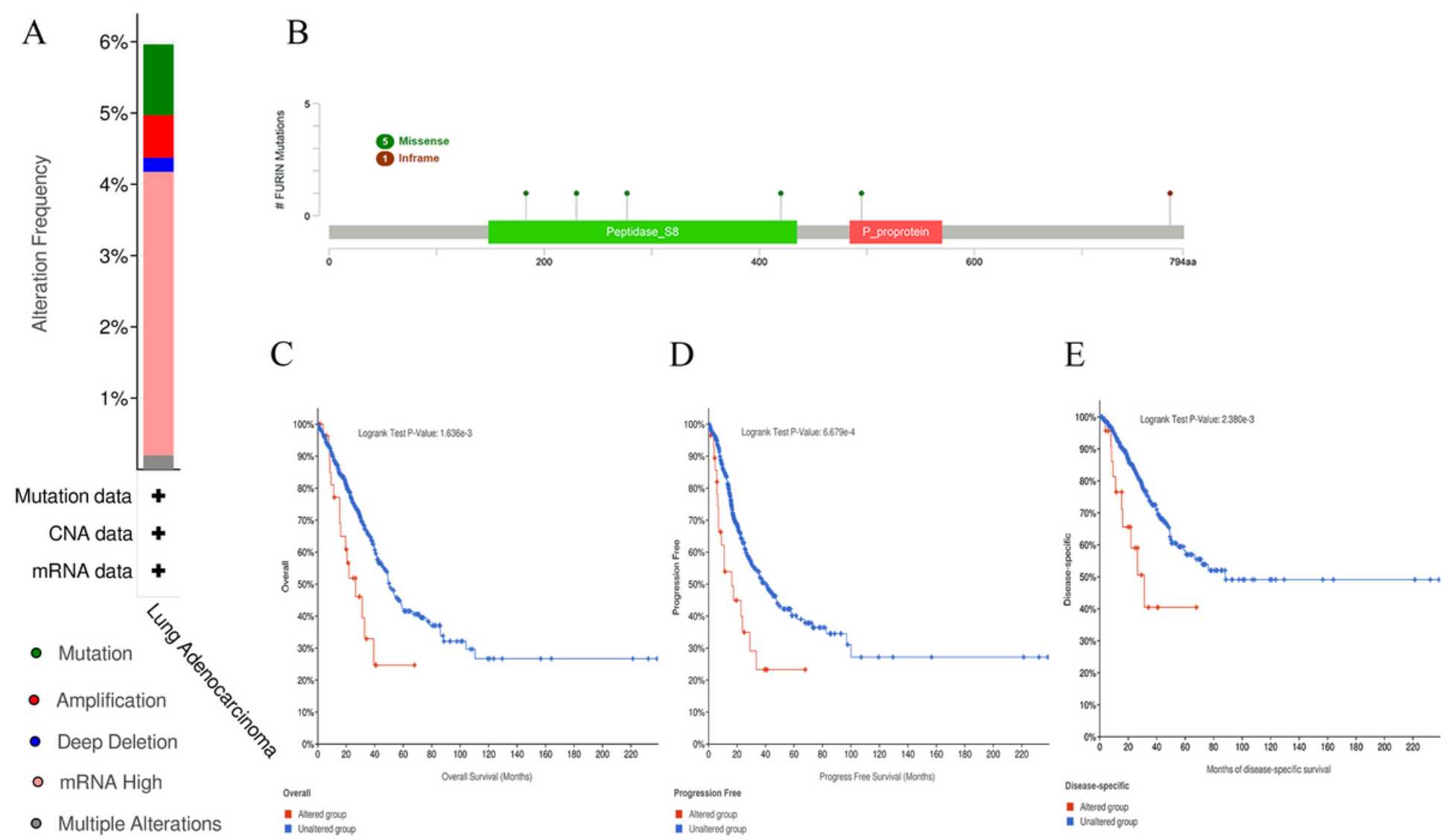

Figure 4

FURIN in LUAD patients changes frequency and type, prognostic condition. (A) FURIN gene changes frequency and type. (B)The types, sites and case number of the FURIN genetic alteration. (C-F) Clinical survival analysis of FURIN-altered LUAD patients.

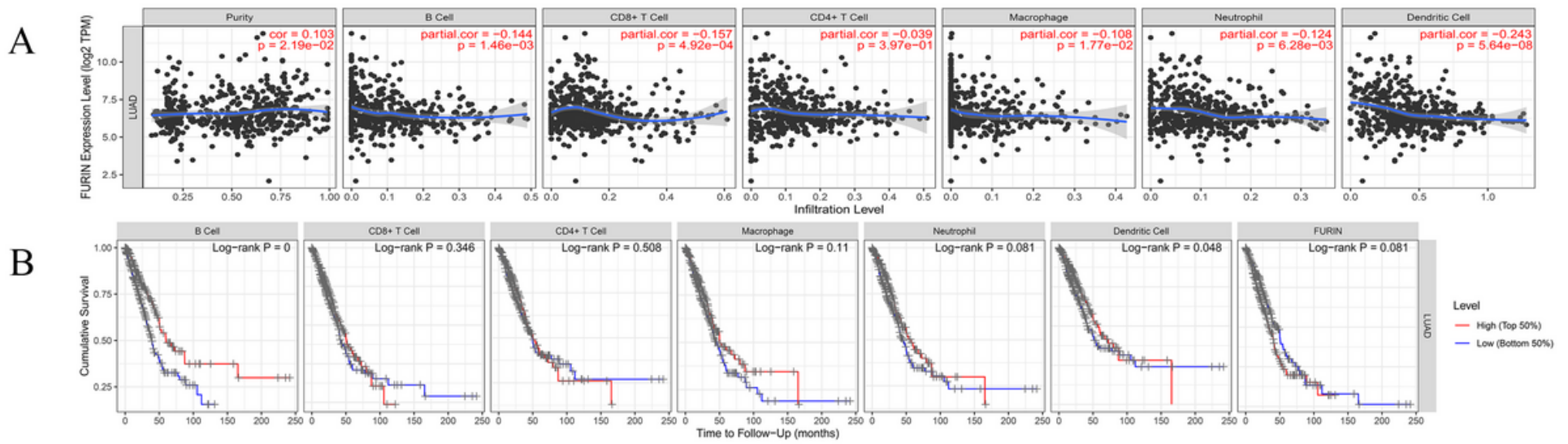

\section{Figure 5}

Correlation analysis of FURIN expression with six immune filtration cells in LUAD. (A) The correlation between FURIN expression and six immuno-permeable cells in TIMER. (B) The overall survival curve of the six immune cells mapped by Kaplan-Meier in TIMER. 
A

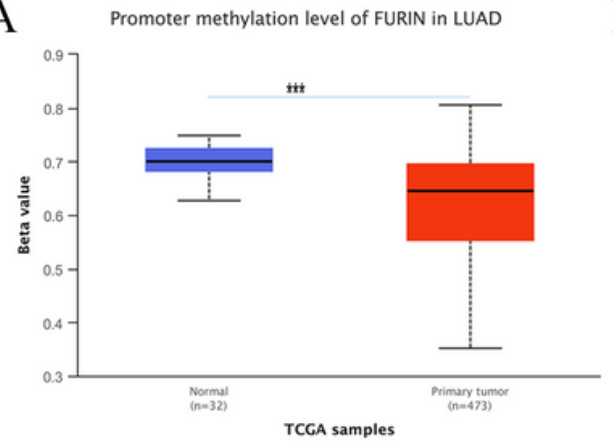

D

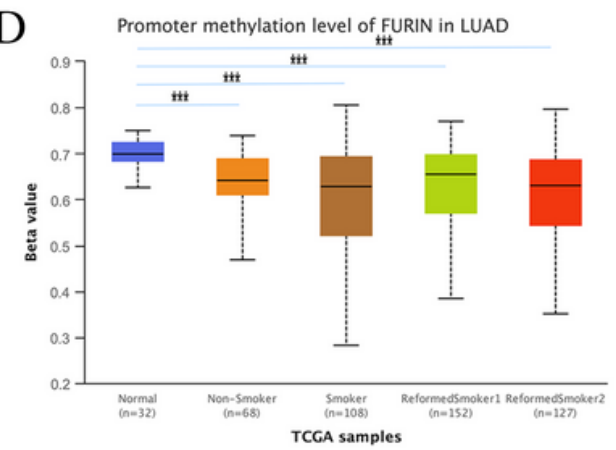

B

Promoter methylation level of FURIN in LUAD

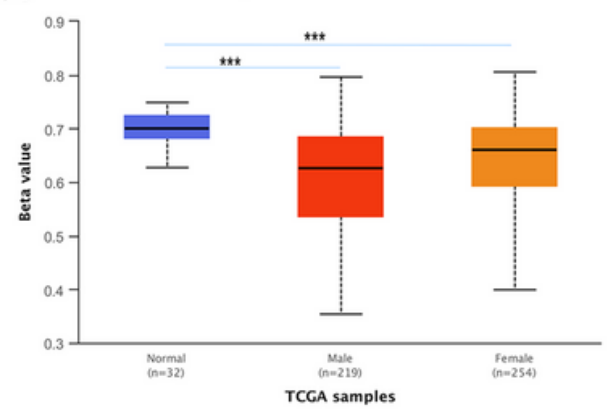

E Promoter methylation level of FURIN in LUAD

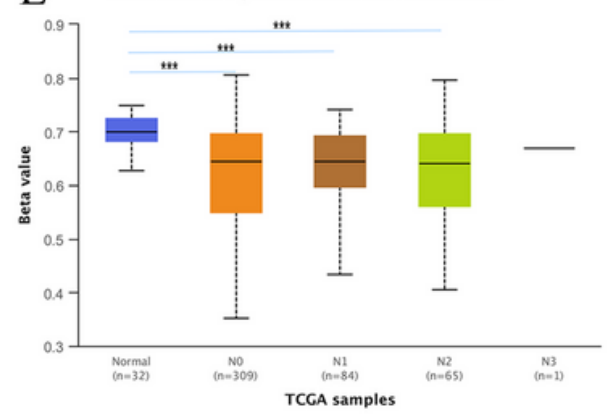

C Promoter methylation level of FURIN in LUAD

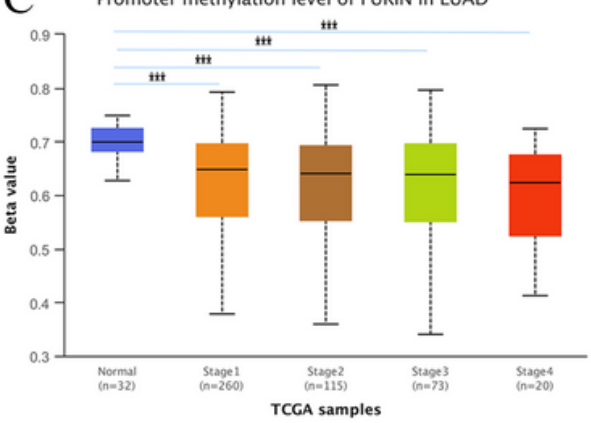

F Promoter methylation level of FURIN in LUAD

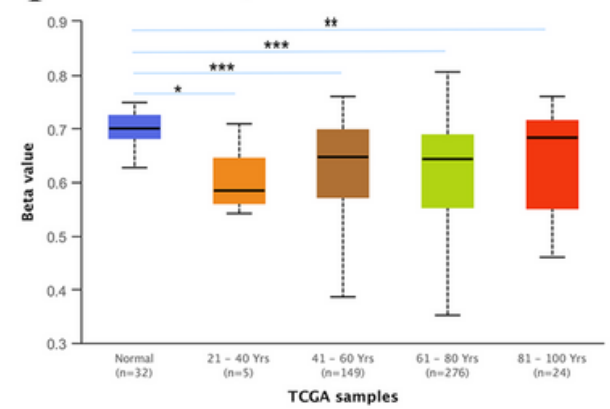

\section{Figure 6}

FURIN expression is associated with the immersion and polarization of macrophages. (A-B) Using EPIC, XCELL studied the correlation between FURIN expression and macrophage immersion levels. (C-D) Using CIBERSOFT, CIBERSOFT-ABS studied the correlation between FURIN expression and M1 macrophage immersion levels. (E-F) Using EPIC, CIBERSOFTABS to study the correlation between FURIN expression and M2 macrophage immersion levels. (G) FURIN expression and M1 macrophage gene marker correlation scattering map. $(\mathrm{H})$ FURIN expression and M2 macrophage gene markers correlation scattering map. 

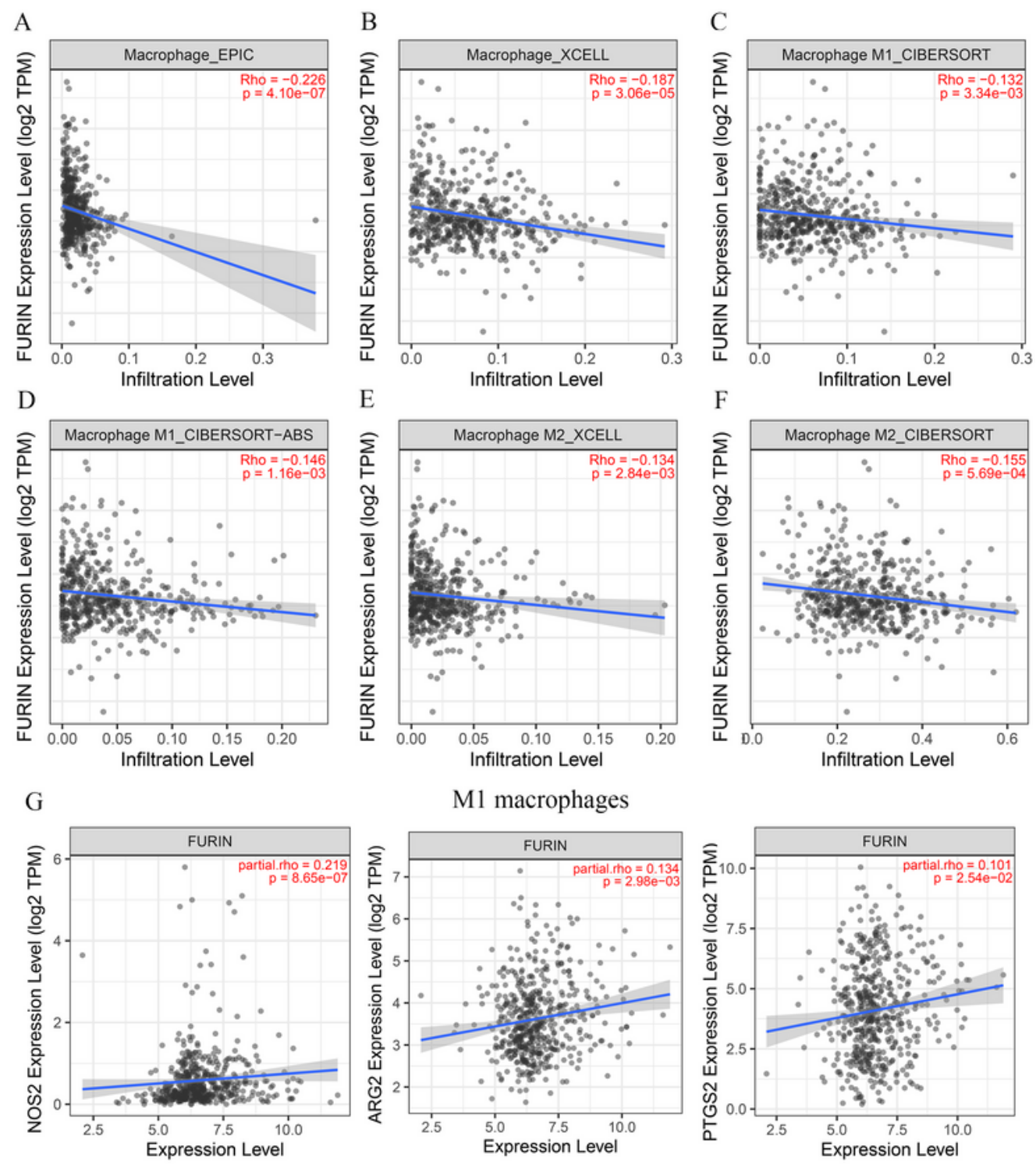

M1 macrophages
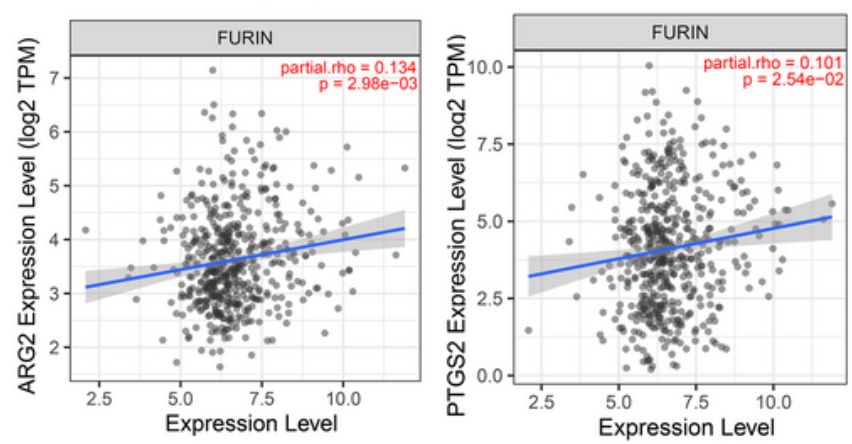

$\mathrm{H}$

M2 macrophages
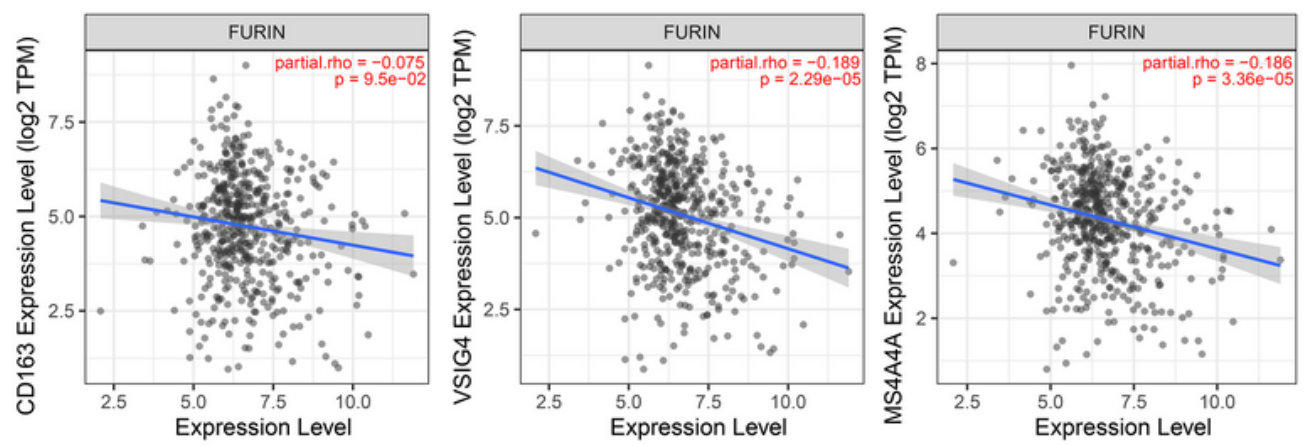

\section{Figure 7}

The level of promoter methylation of FURIN in LUAD. The level of promoter methylation of FURIN is low (A-F). $\left({ }^{*} p<0.05, * * p<0.01\right.$, and $\left.* \star * p<0.001\right)$. 
A

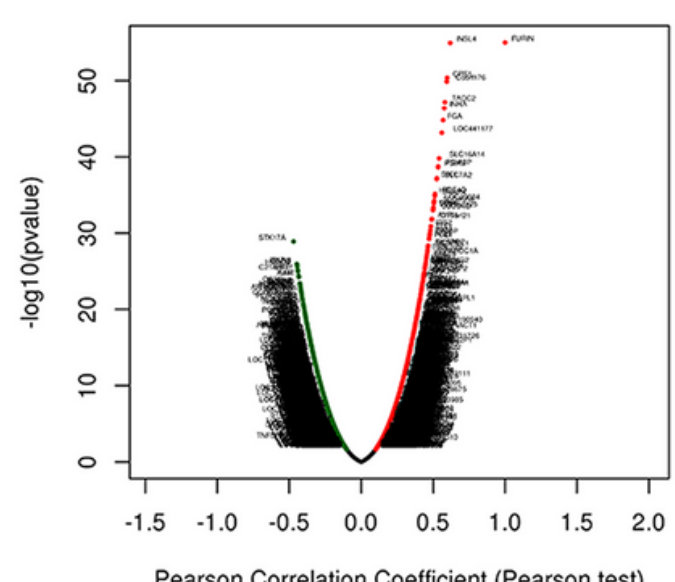

B

Prositively Correlation Significant Genes

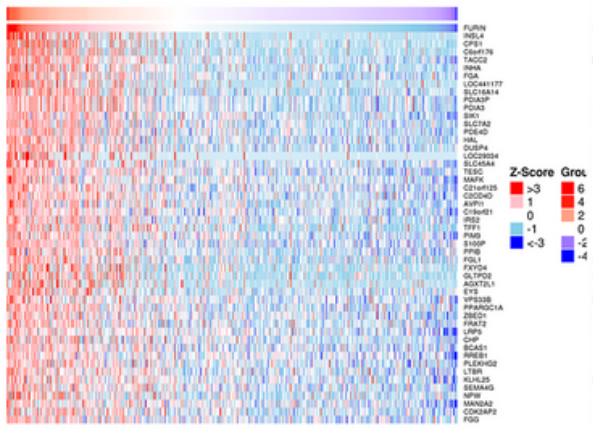

$\mathrm{C}$

Negatively Correlation Signifiant Genes

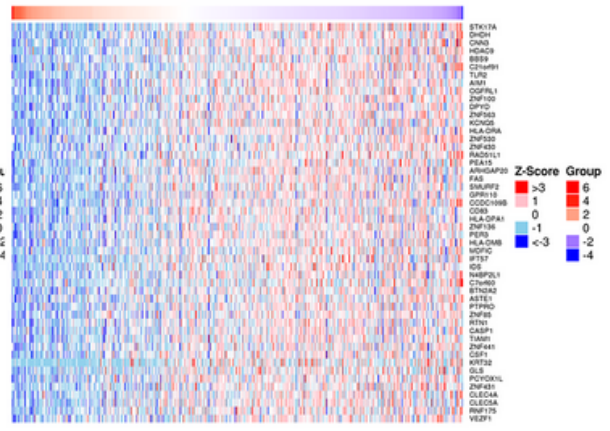

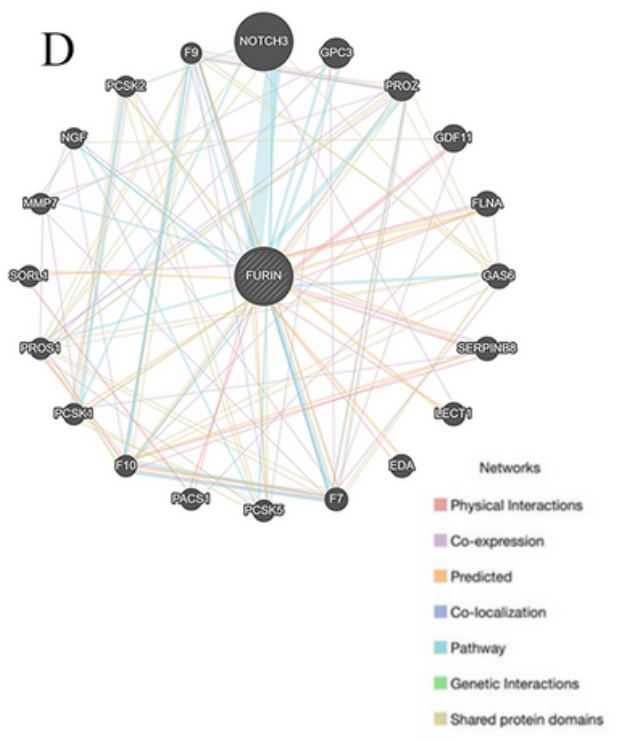

E
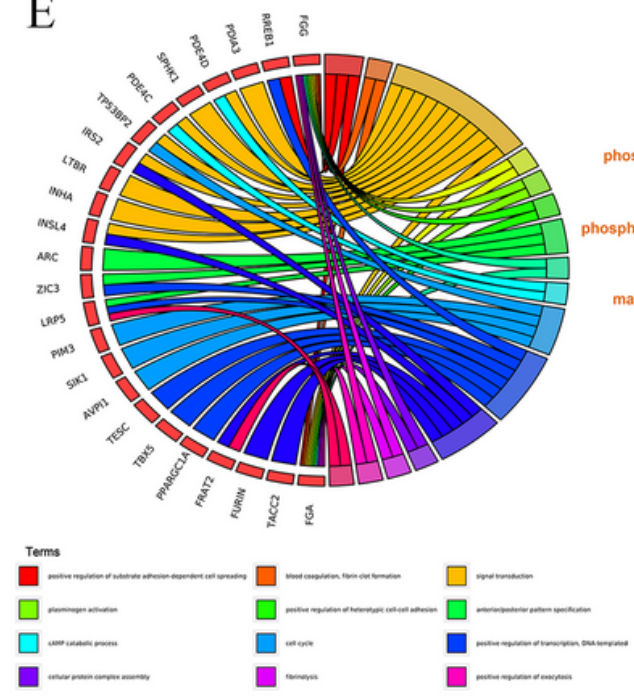

F

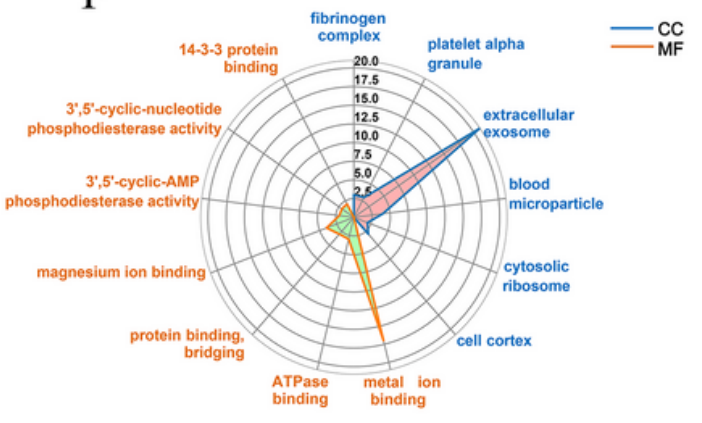

Figure 8

FURIN co-expression gene in LUAD. (A) The global FURIN highly correlated gene identified by the Pearson test in LUAD. Red ang green dots represent positively and negatively significantly correlated genes with FURIN. (B-C) Heatmaps showing the top 50 gene positively and negatively correlation with FURIN in LUAD. (D) PPI network of FURIN in GeneMANIA, different colors of the network edge indicate the bioinformatics methods applied: physical interactions, co-expression, predicted, co-localization, pathway, genetic interactions, and shared protein domains. (E-F) Significantly enriched GO: Biological process (BP), Cell composition (CC), Molecular function (MF). 


\section{GSE44274 \\ 후 Control 후 Treat}

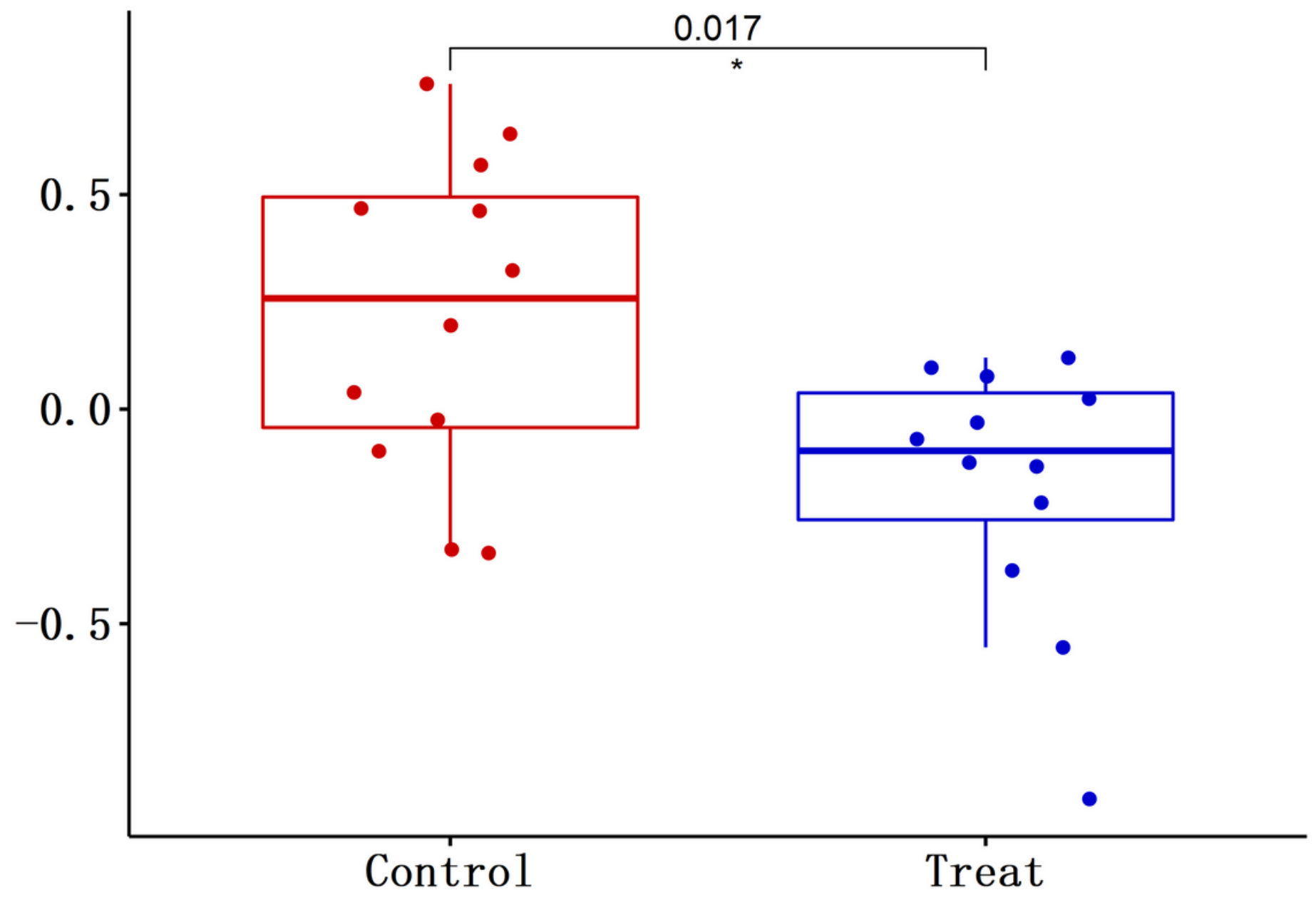

Figure 9

SARS-COV infected the expression levels of FURIN in GSE44274. 